\title{
Design of Carbon Nanotube Antenna in Nanoscale Range
}

\author{
Muna Hajjyahya*, Maen Ishtaiwi*, Jeelan Sayyed, Ayyam Saddouq \\ Department of Physics, An-Najah National University, Nablus, Palestine \\ Email: ^m.hajjyahya@najah.edu, *m.ishtaiwi@najah.edu, jelan.sayed@najh.edu, eng.ayyam1@gmail.com
}

How to cite this paper: Hajjyahya, M., Ishtaiwi, M., Sayyed, J. and Saddouq, A. (2021) Design of Carbon Nanotube Antenna in Nanoscale Range. Open Journal of Antennas and Propagation, 9, 57-64. https://doi.org/10.4236/ojapr.2021.94005

Received: September 28, 2021

Accepted: November 7, 2021

Published: November 10, 2021

Copyright $\odot 2021$ by author(s) and Scientific Research Publishing Inc. This work is licensed under the Creative Commons Attribution International License (CC BY 4.0).

http://creativecommons.org/licenses/by/4.0/

\section{(c) (i) Open Access}

\begin{abstract}
In this paper, new values of efficiency for coated and non-coated carbon nanotube dipole antennas are found at different ranges of length and constant radius to have an effective model in wireless communication technology, biomedical engineering, sensors and solar cells. The main issue is to get matching between the antenna and the feeding source (discreet port). To have the best value of matching impedance by optimization method through entering trials of impedance value the aim of this paper is to have the best result of efficiency in each length. A new value of efficiency is shown for coated carbon nanotube dipole antennas, it is about $59 \%$.
\end{abstract}

\section{Keywords}

Carbon Nanotube, Nanoantenna, Optimization Method

\section{Introduction}

Carbon nanotubes (CNTs) have been investigated as antennas in various areascommunications between Nanodevices and macroscopic world, nano interconnect, fiber communication, aircraft and space communication systems. Researchers assumed that CNTs antennas technology will be at the frontier of scientific research for the next decades, especially in wireless communication technology with nanometer length of CNTs dipole antenna, the electromagnetic (EM) radiation from this antenna is expected to cover a range within terahertz $(\mathrm{THz})$ and optical frequency [1] [2].

CNTs have high electrical properties which make them distinguished from other materials. This assumption was presented, based on the idea that it can radiate as a small Nano-dipole antenna when it is electromagnetically exciting. Single wall carbon nanotubes (SWCNTs) were presented as a theoretical study to 
characterize the $\mathrm{THz}$ antenna based on combining the Boltzmann transport equation and Maxwell's equations with boundary conditions of the electron distribution function [3]. The performance of CNTs dipole antennas has been investigated using comprehensive numerical techniques. In this paper, a virtual CNTs model is developed to be used with commercial software packages to deduce the performance of various CNTs antenna configurations. There are several methods for the investigation of the properties of the CNTs antenna [1].

The complex permittivity approach has been adopted to know the constitutive parameters of the antenna material that will be modeled to become ready for the simulation. Quantitative predictions of the performance of CNTs antenna dipoles, including input impedance, and efficiency, are presented as a function of frequency [4] [5].

New material structure (NMS) is presented to enhance antenna properties consisting of SWCNTs dipole antenna coated by a thin layer (in nm scale) of several materials separately as aluminum or copper. The simulation of the equivalent modeling approach for SWCNTs-composite material is illustrated. The study of their EM properties is very important to offer new material for antenna design at different applications [6]. In order to predict the EM behavior of the SWCNTs-composite material, the important parameters of this material have been extracted. Hence, these parameters are utilized to represent the equivalent modeling approach of SWCNTs [7] [8].

Computer Simulation Technology is a powerful numerical full electromagnetic simulator. Maxwell's equations can be solved numerically inside the structure to make it simpler. The behaviors of electric and magnetic fields within the structure in either time or frequency domain have been contained in CST Studio Suite software. To simulate SWCNTs-composite dipole antenna we used a time domain field.

The trails of having a dipole antenna with the efficiency higher than the copper one have been made by researchers in recent years [1]. Copper dipole antenna does not offer reliable efficiency when it transmits or detects terahertz frequency signal because of skin effect. The idea of using SWCNTs dipole antenna without any skin effect has been established to develop the dipole antenna and have a small size lightweight prototype. But the value of efficiency does not reach the desired goal. However, the previous study has shown that the radiation efficiency of a single-walled carbon nanotube (SWCNT) antenna is very low, which is mainly attributed to the strong retarded surface wave that reduces the radiation resistance. On the other hand, the problem of impedance mismatch is significant if a single CNT is used to build an antenna because its characteristic impedance $(10-100 \mathrm{k} \Omega)$ is extremely large compared to the normal feeding line $(50 \Omega)$ [1]. Because of this, the idea of coating SWCNT has been studied to have dipole antenna works on the high frequency with probable efficiency [9]. Optimization method is the way that has been approved to solve the problem of impedance mismatch. 


\section{Structure of Proposed Composite Material}

Composite material in the 3D EM computer simulation technology (CST). Where, this equivalent model is equivalent bulk material (EBM). The EBM model has the same properties of SWCNTs composite material such as, effective conductivity, complex permittivity, plasma frequency, and relaxation frequency. Therefore, by inserting these material parameters in the available 3D EM simulation software packages, the EBM model can be designed and implemented [7] [9].

Complex permittivity approach had been applied to predict the electromagnetic properties of SWCNT_copper dipole antennas. Through previous approach, Drude dispersion model was adopted and effective parameters were calculated using Equation (2) and Equation (4). The values of plasma frequency and relaxation frequency were calculated as $w_{p}=9.7 \mp 10^{14} \mathrm{rad} / \mathrm{s}, \quad v=6.08 \times 10^{10} 1 / \mathrm{s}$.

\section{Methodology}

In this paper, a new material structure (NMS) is presented to design a dipole antenna for wireless antenna applications at $\mathrm{THz}$ frequency band and fulfill the required enhancement for the antenna properties. The effective model approach will be used for modelling the NMS [9].

Researchers have attempted to produce new materials necessary for designing antennas for future technology. There is an urgent demand for designing and implementing antennas based on new material structures with remarkable properties. To achieve this aim, this work proposes a new material structure based on CNTs material (SWCNTs), due to their rare properties, through the integration of CNTs material with other materials. The CNTs-composite material consists of SWCNT coated by a thin layer of copper to construct (SWCNT-copper) material and SWCNT coated by aluminum to construct (SWCNT-aluminum) material [7] [10]. The main structure of CNTs-composite material (NMS) is illustrated in Figure 1.

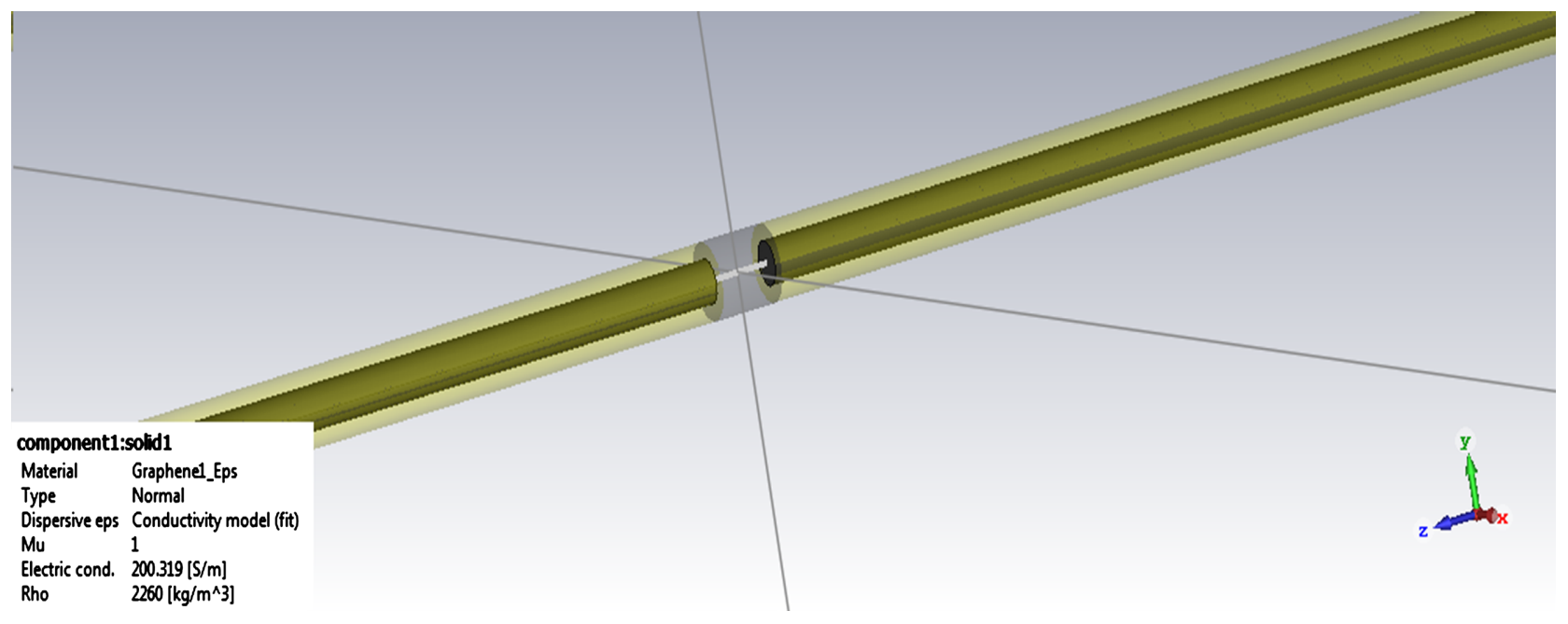

Figure 1. Structure of SWCNTs-composite material (SWCNTs coated by another material). 


\section{Mathematical Analysis of SWCNT-Composite Material}

First step of modeling SWCNTs to create CNTs on software package (CST), the method of defining SWCNTs by using complex permittivity approach in two ways either by Discrete values of and tabulated in dielectric dispersion fit window and computed using MATLAB codes, or by Drude dispersion model have been discussed.

The electrical conductivity is an important property of the NMS (SWCNTcomposite material). Similarly, the influences of the SWCNT and layer of coated material are related significantly to the electrical conductivity of the NMS. Therefore, the estimation of this property is for the new structure.

\section{Drude Dispersion Model}

Mathematical dispersion formulas in Drude Dispersion model have been used to evaluate the material's optical properties by adjusting specific set parameters. Drude's model is based on the kinetic theory of electrons in a metal which assumes that the material has motionless positive ion and non-interacting electron gas using classical mechanical theory of free electron to explain the transport properties of conduction electrons in metals, conductive oxides, and semiconductors.

\section{Simulation Modelling Approach of New Material Structure}

In modern research of antenna applications, the investigation of electromagnetic properties is very important. Complex permittivity approach has been applied to predict the electromagnetic properties of the CNT antennas [5] [8]. Through the previous approach, Drude dispersion model is adopted and effective parameters are calculated. Mathematical dispersion formulas in the Drude Dispersion model have been used to evaluate the material's optical properties by adjusting specific set parameters. The value of epsilon infinity is one for all types of CNTs. The value of plasma frequency for SWCNTs antenna without coating and with coating material is computed from Equations:

$$
\begin{gathered}
w_{p}=\frac{2 e}{\pi r} \sqrt[2]{\frac{V_{f}}{\varepsilon_{0} \hbar}} \\
w_{p}=e^{2} / \pi(r+t)\left[\frac{4 V_{f}+\pi^{2} \hbar M}{\hbar \varepsilon_{0}}\right]^{1 / 2}
\end{gathered}
$$

where, $V_{f}$ is the Fermi velocity for CNTs, $r$ is the radius of CNTs and $t$ is the thickness of coating layer $M=\frac{(R 2-r 2) v \sigma_{\text {coat }}}{e^{2}}, \sigma_{\text {coat }}$ is the conductivity of coating layer [7] [9].

And the collision frequency is considered as the same value of the relaxation frequency for SWCNTs antenna without coating and with coating material computed from equations

$$
\begin{gathered}
v=6 T / r \\
v=6 T /(r+t)
\end{gathered}
$$




\section{Modelling of Antenna Parameters}

\section{Matching Impedance of Antenna Model}

Matching issue between antennas and the feeding source (discreet port) is one of the main problems of SWCNTs dipole antenna and SWCNTs-composite dipole antenna. This matching approach depends on changing the Normalized Fixed Impedance (NFI) of the parameter to balance the effectiveness of the input impedance of these dipole antennas with the internal impedance of the feeding source [4] [7] [11]. Optimization method is the way that had been presented to find the most suitable impedance that matched perfectly to have the most efficient model of nano antenna for four possible lengths.

\section{Simulation Results and Discussions}

\subsection{Effective Permittivity Charts after Defining CNTs at CST}

After defining CNTs through dispersion models, charts of the real and imaginary part of permittivity have appeared, or we can have a permittivity chart through writing equations of permittivity as code on MATLAB. Effective permittivity for SWCNTs-Cu dipole antenna real and imaginary part as a function of frequency have been shown in Figure 2(a) and Figure 2(b). That the value of

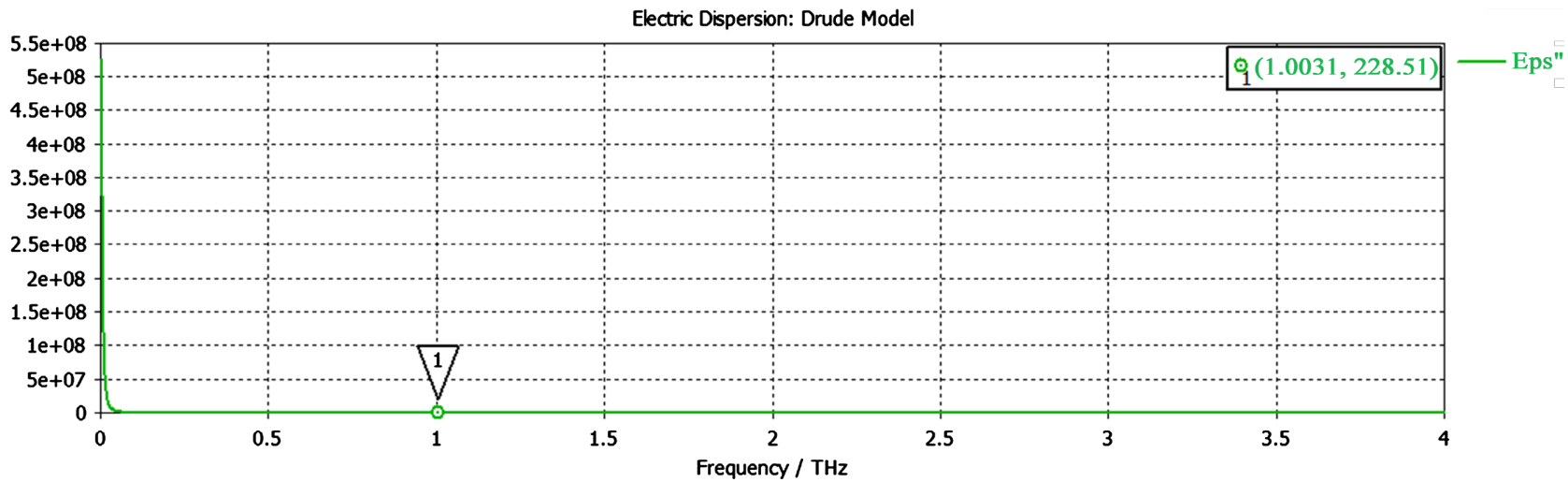

(a)

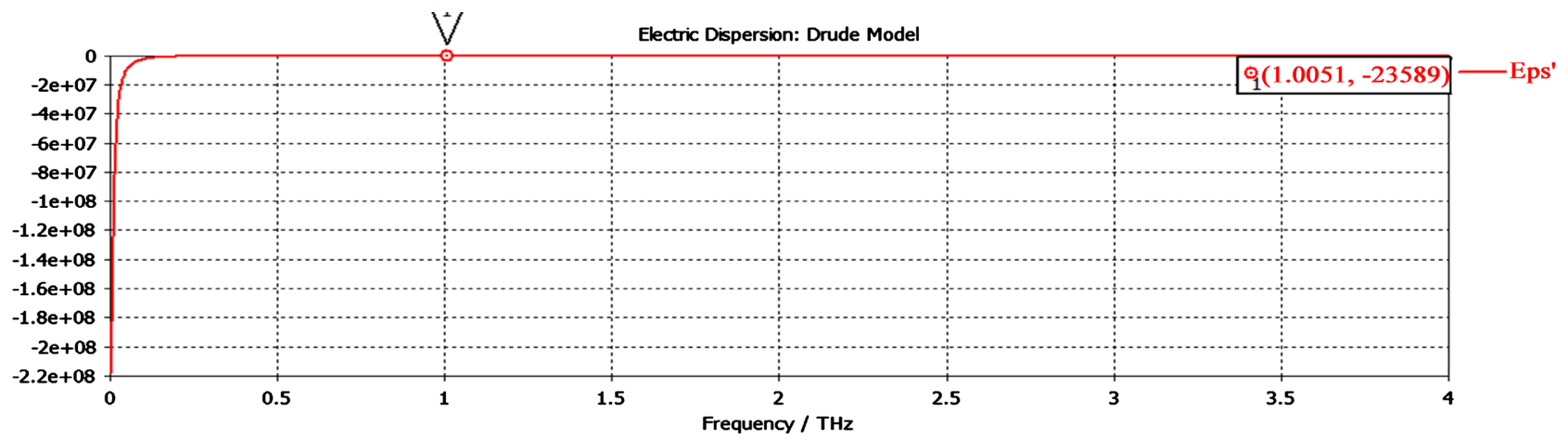

(b)

Figure 2. Effective complex permittivity (a) Real part of complex permittivity as a function of frequency of SWCNTs dipole antenna coated by copper of radius $r=2.71 \mathrm{~nm}$, thickness $2 \mathrm{~nm}$ and length $L=10 \mu \mathrm{m}$. (b) Imaginary part of complex permittivity as a function of frequency of SWCNTs dipole antenna coated by copper of radius $r=2.71 \mathrm{~nm}$, thickness $2 \mathrm{~nm}$ and length $L=10 \mu \mathrm{m}$. 
permittivity (real part) at almost $1 \mathrm{THz}$ has been obtained as 228.51. While the imaginary part of permittivity is equal to 23589 .

\subsection{Efficiency}

The radiation efficiency of SWCNTs antenna had very low values that restricted its application as an antenna in the $\mathrm{THz}$ regime. SWCNTs-composite material is used to increase the radiation efficiency very significantly in the $\mathrm{THz}$ regime. By using the steps followed to define SWCNTs-composite dipole antenna in previous researches. SWCNTs-copper dipole antenna was created and the required simulation steps have been followed [6] [7] [8] [9] [11].

This section presents the simulation results of the efficiency of the proposed material structure NMS (SWCNT-copper) dipole antenna with 10, 20, 30, $40 \mu \mathrm{m}$ length and of $2 \mathrm{~nm}$ copper layer is presented in Figures 3(a)-(d), respectively.

The highest value of radiation efficiency has been obtained in this paper at 30 $\mu \mathrm{m}$ length, and it is equal to $59.14 \%$.

\section{Conclusions}

In this paper, the efficiency of SWCNTs-coated and non-coated dipole antennas as a function of frequency are found at different ranges of length and constant

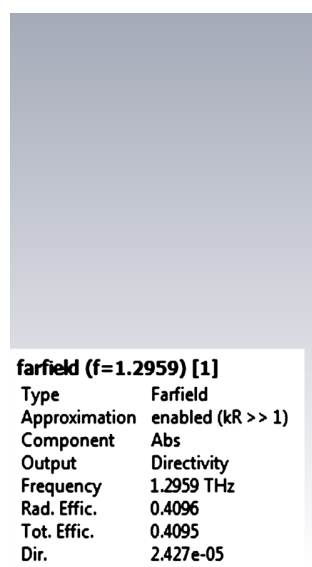
Type Farfield Approximation enabled $(k R>>1)$ Component Abs Output Directivity Frequency $\quad 1.1164 \mathrm{THz}^{2}$ $\begin{array}{ll}\text { Tot. Effic. } & 0.4297 \\ \text { Dir. } & 5.144 \mathrm{e}-06\end{array}$ farfield $(f=1.1164)[1]$ Rad. Effic. $\quad 0.4306$
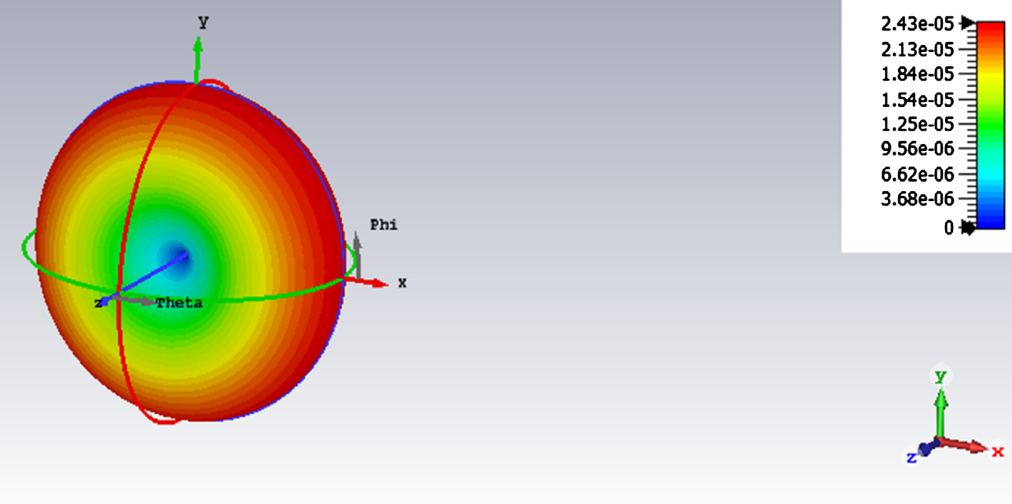

(a)

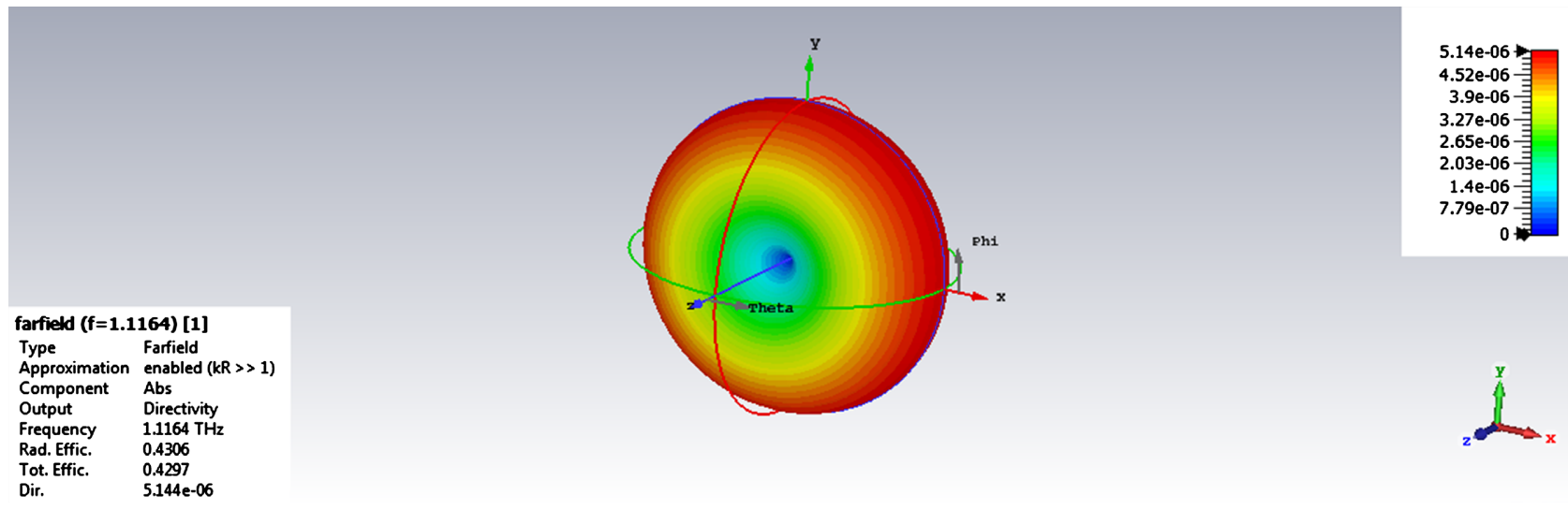

(b) 


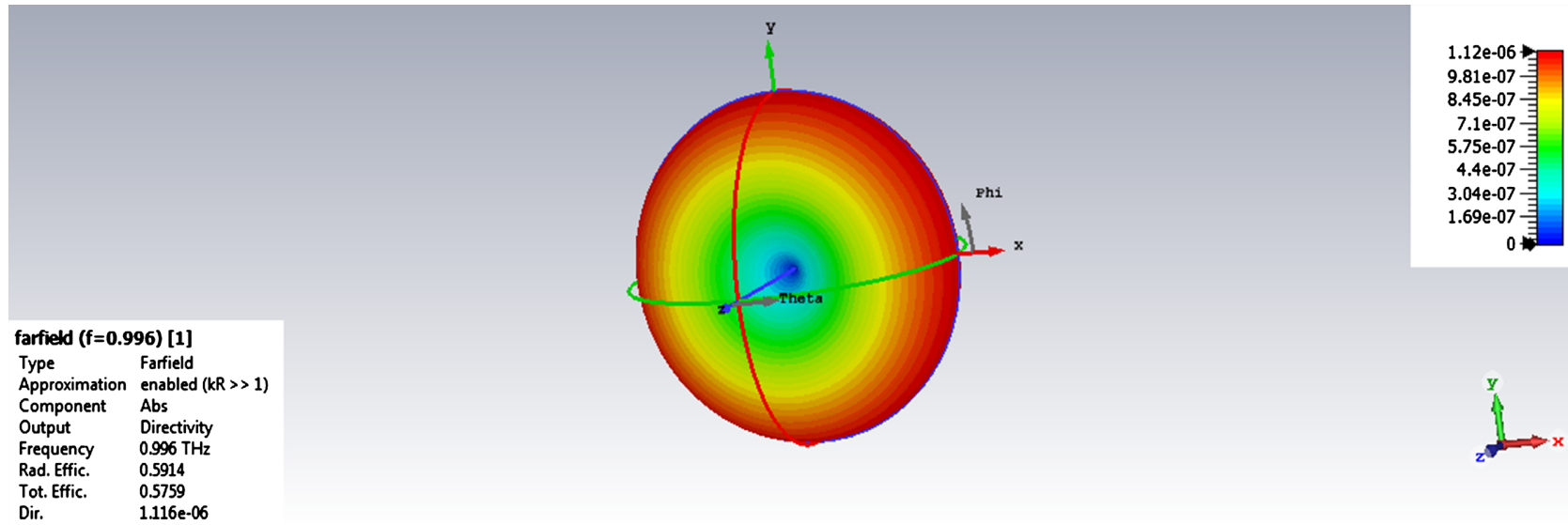

(c)

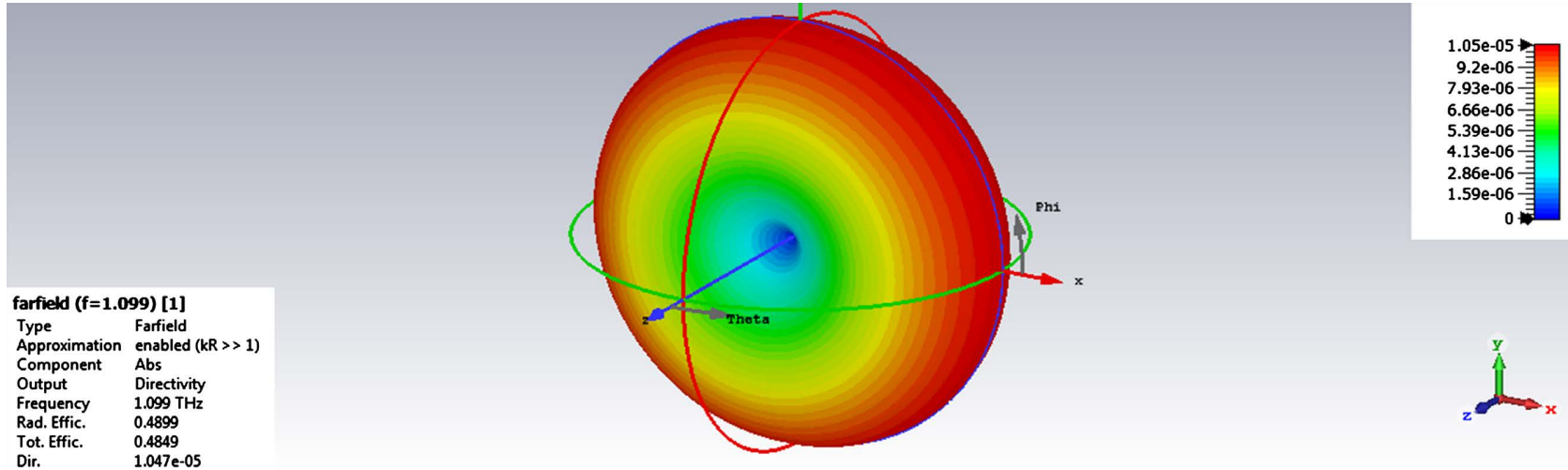

(d)

Figure 3. 3D far field plot of SWCNTs-Cu dipole antenna, where $\mathrm{L}=10,20,30,40 \mu \mathrm{m}$ and radius $2.71 \mathrm{~nm}$, thickness layer $2 \mathrm{~nm}$ of copper.

radius. The result shows new values of efficiency for coated carbon nanotube dipole antenna, it is about $59 \%$ in the case of zero loss and $77 \%$ in the case of loss due to miss matching impedance. In future work, the aspiration is to design a terahertz antenna that will use in biomedical engineering very efficiently to get the devices as small as possible with higher efficiency values and faster data rate in form of $0 \mathrm{D}$ Graphene terahertz antenna that radiated EM waves isotropically.

\section{Conflicts of Interest}

The authors declare no conflicts of interest regarding the publication of this paper.

\section{References}

[1] El-Sherbiny, S.G., Wageh, S., Elhalafawy, S.M. and Sharshar, A.A. (2013) Carbon nanotube Antennas Analysis and Applications. Advances in Nano Research, 1, 13-27. https://doi.org/10.12989/anr.2013.1.1.013

[2] Kempa, K.K., Rybczynski, J. and Huang, Z.P. (2007) Carbon Nanotube as Optical antennae. Advanced Materials, 19, 421-426.

https://doi.org/10.1002/adma.200601187 
[3] Sa'don, S.H., Jamaluddin, M., Kamarudin, M., Ahmad, F., Yamada, Y., Kamardin, K. and Idris, I. (2019) Analysis of Graphene Antenna Properties for 5G Applications. Sensors, 19, 4835. https://doi.org/10.3390/s19224835

[4] Burke, P.J., Li, S. and Yu, Z. (2006) Quantitative Theory of Nanowire and Nanotube Antenna Performance. Institute of Electrical and Electronics Engineers (IEEE), 5, 314-334. https://doi.org/10.1109/TNANO.2006.877430

[5] Jean, R. and Anderson, C. (2005) Determining the Complex Permittivity of Materials with the Waveguide-Cutoff Method. Ph.D. Thesis, Baylor University, Waco, Texas.

[6] Xiaojing, W., Chao, W., Liang, C., Shuit-Tong, L. and Zhuang, L. (2012) Noble Metal Coated Single-Walled Carbon Nanotubes for Applications in Surface Enhanced Raman Scattering Imaging and Photo Thermal Therapy. Journal of the American Chemical Society, 134, 7414-7422. https://doi.org/10.1021/ja300140c

[7] Yaseen, J., Mohamed, A. and Hasliza, R. (2018) Carbon Nanotubes Composite Materials for Dipole Antennas at Terahertz Range. Progress in Electromagnetics Research $M$, 66, 11-18. https://doi.org/10.2528/PIERM17101101

[8] Kadhom, M.J., Aziz, J.S. and Fyath, R.S. (2012) Performance Prediction of Carbon Nanotube Dipole Antenna Using the Complex Permittivity Approach. Journal of Emerging Trends in Computing and Information Sciences (CIS), 3, 1581-1600.

[9] Yaseen, N.J., Malek, M.F. and Hasliza, A.R. (2016) Mathematical Analysis and Modeling of Single Walled Carbon Nanotube Composite Material for Antenna Applications. Progress In Electromagnetics Research M, 45, 59-71. https://doi.org/10.2528/PIERM15091702

[10] Jurn, Y.N., Mahmood, S.A. and Habeeb, I.Q. (2020) Performance Prediction of Bundle Double-Walled Carbon Nanotube-Composite Materials for Dipole Antennas at Terahertz Frequency Range. Progress In Electromagnetics Research M, 88, 179-189. https://doi.org/10.2528/PIERM19101604

[11] Divyaraji, G. and Trushar, P. (2018) $2.45 \mathrm{GHz}$ Antenna Design with Impedance Matching Network: NIBE AB, Markaryd, Sweden. Master Thesis, Halmstad University, Markaryd, Sweden. 